\title{
Um Estudo Preliminar de Ecossistemas de Software na Disciplina Engenharia de Software
}

\author{
Emanuel F. Coutinho' ${ }^{1}$, Italo Santos ${ }^{2}$, Leonardo O. Moreira ${ }^{1}$, Carla I. M. Bezerra ${ }^{3}$ \\ ${ }^{1}$ Instituto UFC Virtual - Universidade Federal do Ceará (UFC) - Fortaleza, CE - Brasil \\ ${ }^{2}$ Instituto de Ciências Matemáticas e de Computação - Universidade de São Paulo \\ (ICMC/USP) - São Carlos, SP - Brasil \\ ${ }^{3}$ Campus Quixadá - Universidade Federal do Ceará (UFC) - Quixadá, CE - Brasil \\ emanuel@virtual.ufc.br, italo.santos@usp.br, \\ leoomoreira@virtual.ufc.br, carlailane@.ufc.br
}

\begin{abstract}
The Software Engineering discipline is commonly taught in several undergraduate courses related to Computing and Engineering. A Software Ecosystem (SECO) refers to a collection of software products with some degree of symbiotic relationship, and may consist of a set of actors interacting as a unit, which in turn interact with a distributed market between software and services. This paper aims to report an experience of the SECO teaching in the Software Engineering discipline in an undergraduate course. It was noticed by the initial results that the teaching of SECO in the discipline could add value to the other areas of Software Engineering. Also in this work, we propose a tool to support the teaching of ECOS.
\end{abstract}

Resumo. A disciplina Engenharia de Software é comumente lecionada em diversos cursos de graduação relacionados à Computação e Engenharias. Um Ecossistema de Software (ECOS) se refere a uma coleção de produtos de software com algum determinado grau de relacionamento simbiótico, podendo consistir de um conjunto de atores interagindo como uma unidade, que por sua vez interagem com um mercado distribuído entre software e serviços. Este trabalho tem como objetivo relatar uma experiência do ensino de ECOS na disciplina de Engenharia de Software em um curso de graduação. Percebeu-se pelos resultados iniciais que o ensino de ECOS na disciplina pode agregar valor às demais áreas da Engenharia de Software. Também neste trabalho propomos uma ferramenta para apoiar o ensino de ECOS.

\section{Introdução e Motivação}

A disciplina Engenharia de Software é comumente lecionada em diversos cursos de graduação relacionados à Computação e Engenharias de maneira geral. Com a evolução da tecnologia da informação e comunicação, alinhada com as necessidades de diversas áreas, o desafio de desenvolver software se torna ainda maior. Além disso, diante de um cenário cada vez mais competitivo entre empresas que desenvolvem software, a qualidade de software é um atributo essencial [Jorge et al. 2015], sendo necessário conhecer melhor ainda elementos não essencialmente de software, mas também de hardware e de partes interessadas. 
VII Congresso Brasileiro de Informática na Educação (CBIE 2018)

Anais do XXIV Workshop de Informática na Escola (WIE 2018)

A criação de um novo produto de software frequentemente envolve grandes equipes de especialistas colocando em prática muitas habilidades, a um custo alto de gerenciamento. Muitas vezes a disciplina de Engenharia de Software conta com uma ampla quantidade de tópicos teóricos que são melhores fixados na prática [Portela et al. 2017]. O desenvolvimento de software necessita de muitas habilidades, como definição de projetos, gerenciamento, programação, validação, análise, estudo dos usuários, documentação, integração de sistemas e técnicas específicas.

Nesse contexto, surge a ideia de conhecer o que é um Ecossistema de Software (ECOS). Um ECOS pode ser referir a uma coleção de produtos de software com algum determinado grau de relacionamento simbiótico [Messerschmitt e Szyperski 2003]. Um ECOS também pode consistir de um conjunto de atores interagindo como uma unidade, que por sua vez interagem com um mercado distribuído entre software e serviços, juntamente com as relações entre as mais variadas entidades [Jansen et al. 2009a]. Estas relações são frequentemente suportadas por uma plataforma tecnológica ou por um mercado comum e realizadas pela troca de informação, recursos e artefatos.

Em geral, disciplinas que alinham a teoria à prática são difíceis de se conduzir devido à necessidade de se tratar fatores técnicos (linguagens de programação, ferramentas, componentes etc.) e humanos (comunicação, gestão, disponibilidade etc.) ao mesmo tempo [Coutinho et al. 2016]. Além disso, a habilidade e experiência do professor em integrar fatores teóricos e práticos também influencia muito na qualidade do aprendizado. De maneira geral, disciplinas de cursos de graduação mais técnicos tendem a possuir uma abordagem curricular mais orientada a produção e tecnologias [Coutinho e Moreira 2017]. Geralmente, cursos mais tradicionais da área de Computação terminam por produzir diferentes tipos de softwares. Devido à interdisciplinaridade das áreas, muitas vezes existem processos de desenvolvimento totalmente diferentes, o que requer interações com os mais variados tipos de perfis e diferentes fornecedores. Nesse contexto, a qualidade do ensino e a compreensão de conteúdos e temas da Engenharia de Software impacta diretamente na melhoria do processo de desenvolvimento de software, podendo apoiar a busca por soluções de problemas e deficiências da indústria de software [Santos et al. 2014].

O curso de graduação em Sistemas e Mídias Digitais (SMD), da Universidade Federal do Ceará (UFC), tem como objetivo a formação de profissionais com conhecimentos em duas grandes áreas principais, Sistemas Multimídia e Mídias Digitais. Esses conhecimentos conjuntos contribuem para novos perfis de profissionais que possam atuar no desenvolvimento de mídias digitais e sistemas multimídia, tais como: sistemas web, dispositivos móveis, jogos digitais, design multimídia e animações gráficas. Além disso, o curso possui características de interdisciplinaridade, contendo em seu corpo docente professores das áreas de Computação, Design Digital, Educação e Comunicação. Assim, estudando os conceitos de ECOS em um curso com tais características reforçaria ainda mais a formação do discente.

Este trabalho tem como objetivo relatar uma experiência do ensino de Ecossistemas de Software (ECOS) na disciplina de Engenharia de Software em um curso de graduação. Além disso, uma ferramenta para o ensino de ECOS é proposta. O restante do artigo está dividido nas seguintes seções: o referencial teórico, a metodologia aplicada ao trabalho, resultados e análises, e por fim, conclusões e trabalhos futuros. 
VII Congresso Brasileiro de Informática na Educação (CBIE 2018)

Anais do XXIV Workshop de Informática na Escola (WIE 2018)

\section{Ecossistema de Software (ECOS)}

Um Ecossistema de Software (ECOS) é uma metáfora na Engenharia de Software que tem sido largamente empregada no entendimento da dinâmica de uma rede de suprimento de software, centradas em uma plataforma de software. Existem diversas definições para ECOS na literatura. A definição mais citada foi introduzida em [Jansen et al. 2009b], onde os autores definem que um ECOS é um conjunto de negócios funcionando como uma unidade e interagindo com um mercado compartilhado de software e serviços, juntamente com as relações entre eles, frequentemente apoiados por uma plataforma ou mercado tecnológico comum e operando através da troca de informações, recursos e artefatos.

Um ECOS é uma interação de um conjunto de atores sobre uma plataforma tecnológica comum, resultando em um número de soluções ou serviços de software [Manikas e Hansen 2013]. Cada ator é motivado por um conjunto de interesses ou modelos de negócio e está conectado aos demais atores e ao ECOS como um todo por meio de relacionamentos simbióticos. Por sua vez, a plataforma tecnológica está estruturada para permitir o envolvimento e a contribuição dos diferentes atores. A metáfora do ecossistema destaca os atores externos e/ou desconhecidos que contribuem para evoluir a plataforma de software comum, modificando a cadeia de valor tradicional e centrada na organização para uma rede de fornecimento de software, onde vários componentes desenvolvidos em diferentes plataformas coexistem e afetam negócios de empresas que realizam transações financeiras [Boucharas et al. 2009].

Uma maneira de formalizar a modelagem de um ECOS foi proposta em [Boucharas et al. 2009], utilizando a estratégia da SSN (Software Supply Network Rede de Suprimento de Software). Como uma das notações de modelagem ECOS mais famosas, o SSN é uma série de software, hardware e organizações de serviços ligados, que cooperam para atender às demandas do mercado [Costa et al. 2013], possuindo uma série de elementos chave. Esses elementos ajudam a representar os principais atores e sua interação dentro de um ecossistema. A Figura 1 exibe os elementos utilizados na notação SSN para a modelagem de um ECOS.

\begin{tabular}{|c|l|}
\hline $\begin{array}{c}\text { Empresa de } \\
\text { Interesse }\end{array}$ & $\begin{array}{l}\text { Empresa de Interesse: Distribui o produto no modelo comercial } \\
\text { definido para o ambiente. Pode ser o produto em si. }\end{array}$ \\
\hline Fornecedor & $\begin{array}{l}\text { Fornecedor: Fornece um ou mais produtos ou serviços } \\
\text { necessários. }\end{array}$ \\
\hline Cliente & $\begin{array}{l}\text { Cliente: Elemento que direta ou indiretamente, adquire ou } \\
\text { utiliza o produto. }\end{array}$ \\
\hline Intermediário & $\begin{array}{l}\text { Intermediário: Atores que atuam como intermediários entre } \\
\text { dois elementos. Ex: distribuidores, revendedores, etc. }\end{array}$ \\
\hline Cliente do & $\begin{array}{l}\text { Cliente do Cliente: Um cliente pode ter seus próprios clientes } \\
\text { com um produto ou serviço direto ou indiretamente da } \\
\text { empresa de interesse. Ex: suporte ao produto, atualizações, etc. }\end{array}$ \\
\hline Agregador & $\begin{array}{l}\text { Agregador: Empresas, produtos ou serviços que operam entre } \\
\text { dois agentes para agregar valor a um produto ou serviço. } \\
\text { Também pode distribuí-lo ou revendê-lo. }\end{array}$ \\
\hline$X . Y$ & $\begin{array}{l}\text { Relacionamento Comercial: Representa um artefato ou fluxo de } \\
\text { serviço de um ator para outro. Pode ser um dado, um software, } \\
\text { dinheiro, serviços, etc. }\end{array}$ \\
\hline & $\begin{array}{l}\text { Fluxo: Conecta dois atores. Um relacionamento pode ser } \\
\text { complexo, constituindo muitos fluxos de direções arbitrárias. }\end{array}$ \\
\hline
\end{tabular}

Figura 1. Notação SSN [Boucharas et al. 2009] com a extensão de [Costa et al. 2013] 
VII Congresso Brasileiro de Informática na Educação (CBIE 2018)

Anais do XXIV Workshop de Informática na Escola (WIE 2018)

\section{Metodologia}

A metodologia aplicada a este trabalho consistiu nas seguintes etapas: (i) apresentação dos conceitos de ECOS em sala de aula; (ii) exemplificação de um ECOS real; (iii) execução de uma atividade de fixação; (iv) execução da atividade do projeto final; (v) coleta dos dados; e (vi) consolidação e análise dos dados.

A apresentação de conceitos relacionados a ECOS ocorreu durante a aula, consistindo inicialmente da motivação, definições variadas, tipos de ecossistemas, benefícios, papéis, dimensões, notação SSN e exemplos. Nessa mesma aula, um exemplo real de ECOS, para o ambiente de e-learning SOLAR [Coutinho et al. 2017a] foi apresentado e discutido com os alunos. Assim, os alunos entenderiam os conceitos e a utilidade de um ECOS com algo mais próximo da realidade deles.

A atividade de fixação foi enviada aos alunos para ser executada em casa. Ela consistiu da descrição de um ECOS para um contexto de interesse do aluno, da maneira que ele achasse melhor. O ideal era utilizar a notação SSN, mas a representação foi livre, desde que o ECOS atendesse às seguintes restrições: funcionar em torno de uma plataforma central, ser um ambiente que contenha elementos de aplicações multimídia, possuir uma imagem que representasse o ECOS, conter pelo menos elementos de hardware, software, clientes, fornecedores, sociedade/comunidade de usuários e desenvolvedores. A atividade do projeto final da disciplina (descrição de um ECOS para a aplicação do projeto final) consistiu da elaboração de uma imagem que representasse $o$ ECOS em questão, com alguns comentários sobre as relações do ambiente, para melhor contextualizar. Nesse exercício, a notação SSN tinha sua utilização obrigatória.

A tarefa de coleta de dados ocorreu após a realização das atividades pelos alunos, seguida de sua consolidação para identificação de pontos de destaque nos comentários dos alunos e dos modelos gerados, e por fim, a análise final.

\section{Resultados e Análises}

Na turma da disciplina de Engenharia de Software no semestre 2017.2, 17 alunos estavam matriculados no início do semestre. Para o trabalho final, equipes foram compostas, sendo no total de 5 equipes, variando de 3 a 5 alunos.

\subsection{Aula de ECOS e ECOS SOLAR}

Inicialmente, uma aula no estilo expositiva foi repassada aos alunos, com a apresentação de slides relacionados ao assunto. Sua intenção foi a transmissão das definições, dos conceitos relacionados e os tipos de ECOS, assim como um momento de discussão conceitual. Benefícios e exemplos também foram comentados. Após a apresentação dos conceitos, mostrou-se um pouco da notação SSN, só para direcionar para alguns elementos que seriam interessantes de serem identificados em uma modelagem de ECOS. E por fim, como estudo de caso, utilizou-se a modelagem do ECOS SOLAR [Coutinho et al. 2017a] como um exemplo, para se discutir as relações entre fornecedores, plataforma central, intermediários e clientes.

O ambiente virtual de aprendizagem (AVA) SOLAR (Sistema Online de Aprendizagem) é uma aplicação web cujo modelo de participação é orientado ao professor e ao aluno, possibilitando a publicação de cursos e interação com os mesmos 
VII Congresso Brasileiro de Informática na Educação (CBIE 2018)

Anais do XXIV Workshop de Informática na Escola (WIE 2018)

[Coutinho et al. 2013]. Ele foi projetado para possibilitar a criação de um espaço virtual para ser utilizado por cursos presenciais ou semipresenciais, servindo como ponto de convergência para a criação do que se está chamando de Blended Education, ou seja, a mescla de características de ambas as modalidades de educação, para formação de um novo modelo educacional que utiliza fortemente Tecnologias da Informação e Comunicação [Coutinho et al. 2017a].

A Figura 2 representa o modelo na notação SSN para o ECOS SOLAR, com seus elementos sendo o próprio AVA SOLAR a plataforma central, seus fornecedores, sejam software livre ou não, seus clientes, os componentes intermediários e agregadores e relacionamentos.

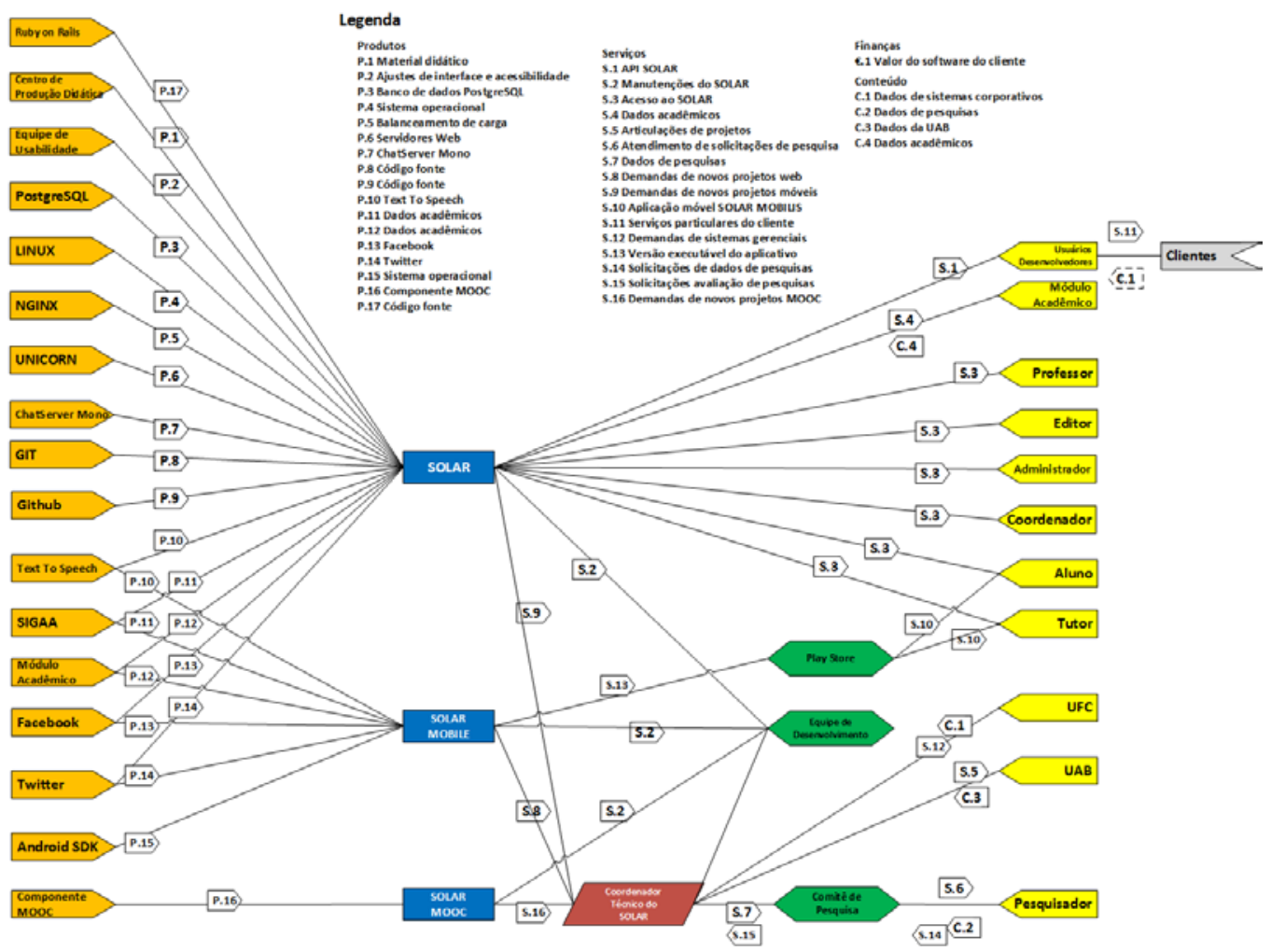

Figura 2. Modelo do ECOS SOLAR com a notação SSN [Coutinho et al. 2017a]

\subsection{Atividade de Fixação e Atividades do Trabalho Final}

A atividade de fixação foi executada de maneira individual. Quatro trabalhos foram analisados, cada um deles com características diferentes, e imagens que representavam o ECOS também em formatos diferentes. Como destaques desta atividade, percebeu-se que todos os alunos descreveram a plataforma central. Este foi o conceito que mais se destacou nas discussões em sala de aula. As plataformas descritas foram variadas, tais como: uma aplicação de interface cérebro computador, jogos digitais, aplicações web e móvel. Também foi comentado sobre linguagens de programação, ferramentas de desenvolvimento e comunidades de desenvolvedores, comuns a ECOS, que produzem novas aplicações, gerando diferentes serviços, às vezes para produtos variados.

A integração com aplicativos de diferentes empresas também foi eventualmente citada. Por fim, a interação dos tipos de usuários e importância das redes sociais para 
VII Congresso Brasileiro de Informática na Educação (CBIE 2018)

Anais do XXIV Workshop de Informática na Escola (WIE 2018)

um ECOS foi discutido como um fator que não necessariamente é software desenvolvido para a plataforma central, mas é um elemento necessário para a divulgação, comunicação e ampliação dos negócios. A Figura 3 exibe alguns modelos desenvolvidos pelos alunos, onde foram desenvolvidos modelos em contextos variados. A notação SSN não necessariamente foi utilizada para sua elaboração.
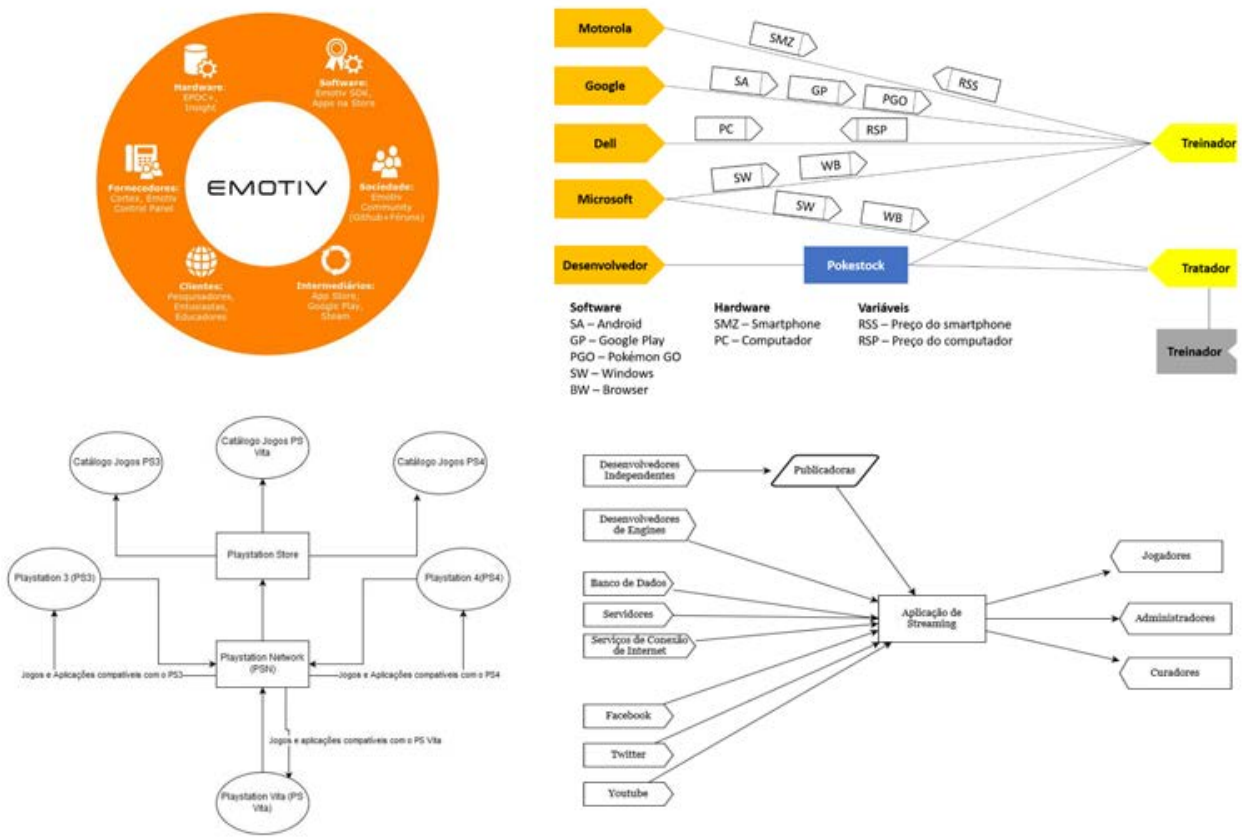

Figura 3. Exemplos de modelos construídos pelos alunos no exercício individual

O trabalho final da disciplina foi realizado em equipe. Este trabalho foi elaborado de maneira progressiva ao longo do semestre, então era de se esperar que os modelos fossem mais detalhados, o que se confirmou na maioria dos casos. A última etapa desse trabalho foi elaborar um diagrama que representasse o ECOS do projeto. Esses ECOS estão exemplificados na Figura 4. Todos os projetos deveriam utilizar a notação SSN. Como não havia ferramenta conhecida que facilitasse a manutenção de modelos, os alunos em geral utilizaram editores gráficos comuns para a criação dos elementos da notação e seus relacionamentos, o que provocava certo desvio em termos de formato, mas nada que atrapalhasse o entendimento e a representação do ECOS.

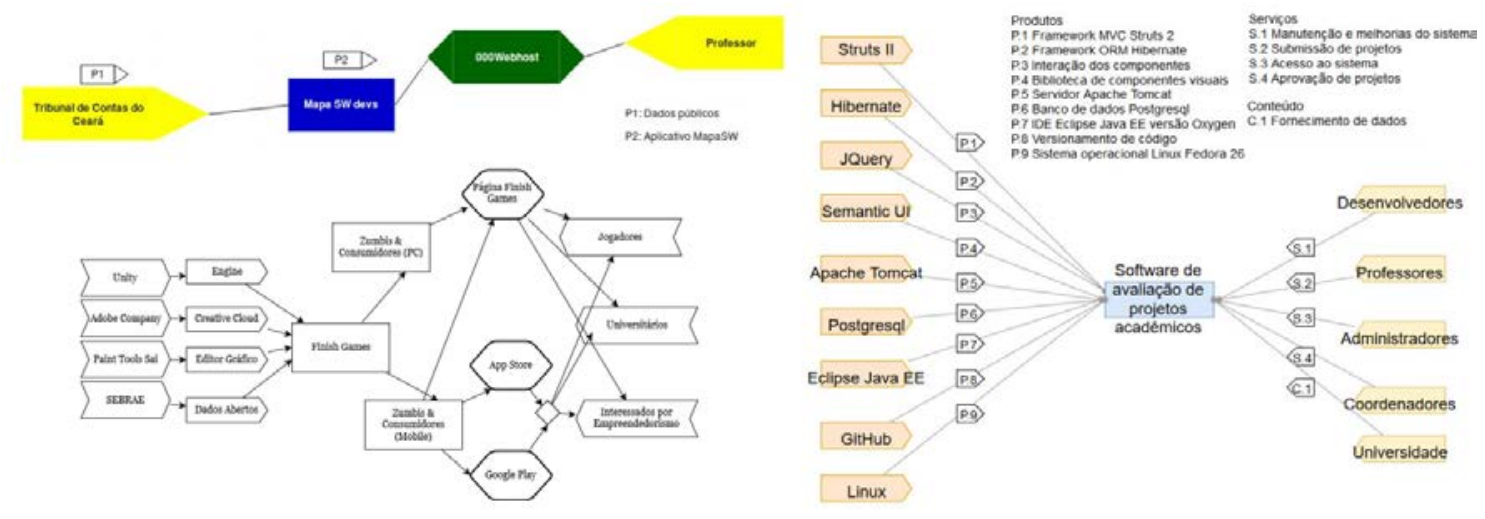

Figura 4. Exemplos de modelos construídos pelos alunos no trabalho final 
VII Congresso Brasileiro de Informática na Educação (CBIE 2018)

Anais do XXIV Workshop de Informática na Escola (WIE 2018)

\subsection{Análise dos Resultados}

Percebe-se pelos modelos gerados nas Figuras 3 e 4 que a ideia central de ECOS foi entendida pelos alunos. Como o foco não era utilizar a notação corretamente, e sim representar um ECOS de qualquer maneira, os alunos atingiram esse objetivo.

Uma percepção do professor da disciplina após a apresentação dos conceitos de ECOS e alguns exemplos é que os alunos tiveram uma noção mais abrangente de todo um ambiente de desenvolvimento de software, de fornecedores, de clientes, e que a qualquer momento pode surgir novos papéis nesse ecossistema, como concorrentes e “clientes de clientes". Outra percepção foi que os alunos começaram a pensar fora do ambiente de projeto, refletindo de maneira mais global, analisando os impactos das diversas partes envolvidas direta ou indiretamente no desenvolvimento de um software.

Em relação aos exercícios individuais dos alunos, as análises se mostraram superficiais, provavelmente devido ao primeiro contato com ECOS. No entanto, as modelagens desenvolvidas mostraram a experiência e o conhecimento dos alunos sobre a plataforma selecionada de maneira geral.

Um destaque nos trabalhos finais, que por terem sido desenvolvidos desde o início do semestre possibilitaram aos alunos mais conhecimento sobre a plataforma central utilizada, foi o comentário sobre a importância dos relacionamentos, principalmente com os distribuidores de softwares que serão utilizados durante o desenvolvimento, e os intermediários responsáveis pela distribuição. Muitos dos distribuidores de software trabalham com plataformas variadas, como dispositivos móveis (Google Play e App Store) e computadores pessoais com sistemas operacionais diferentes, e isso tem um grande impacto no ciclo de vida do desenvolvimento de software e impacto direto no modelo de negócio adotado pela plataforma central.

Uma dificuldade também apontada em [Coutinho et al. 2017b] é a carência de ferramentas de modelagem de ECOS. Ainda é muito livre sua representação, então qualquer notação (diagrama de classes, fluxogramas, imagens arquiteturais etc.) é utilizada para a representação e modelagem de um ECOS. Nem todos os alunos seguiram a notação apresentada (SSN), e alguns comentaram que ela era confusa, que não colaborava para o que eles pretendiam exibir do ecossistema selecionado por eles. Há então uma necessidade de metodologia na modelagem de ECOS. Neste ponto, sugere-se que haja um investimento da comunidade de ECOS na padronização de como se modelar ecossistemas. Isso promoveria um desenvolvimento de notações mais adequadas, ferramentas e facilitaria a comparação entre ECOS.

Como o curso de graduação possui uma filosofia interdisciplinar, o ECOS aparentou ter sido uma boa maneira de descrever visualmente a interdisciplinaridade que pode existir no desenvolvimento de software, quando aplicável. Os diversos perfis de fornecedores e clientes, as diversas partes interessadas e produtos gerados, todos esses elementos podem ser dispostos em uma só imagem, dando uma visão global de um ECOS com características interdisciplinares.

Percebeu-se pelos trabalhos individuais e em equipe que alguns dos modelos foram relacionados a jogos digitais, como plataformas de videogames, empresas de desenvolvimento de jogos, e propostas individuais dos alunos. Isso abre precedência para uma possível linha de pesquisa de ECOS e jogos digitais. Uma ideia seria a 
VII Congresso Brasileiro de Informática na Educação (CBIE 2018)

Anais do XXIV Workshop de Informática na Escola (WIE 2018)

modelagem de ECOS de grandes empresas de desenvolvimento de jogos, pois como normalmente um jogo digital está em diversas plataformas (diferentes consoles de videogames, de dispositivos móveis e de PCs), as relações entre fornecedores, desenvolvedores, produtos e comunidade são bastante variadas e voláteis, isto seria uma grande oportunidade de pesquisa na área de ECOS.

Por fim, algumas melhorias para a disciplina foram sugeridas pelos alunos e percebidas pelo professor: mais exemplos reais de ECOS, de áreas diversas; carga horária maior para o ensino de ECOS; práticas de modelagem de ECOS em laboratório; e antecipação do assunto ECOS mais para o início do semestre. A necessidade de possuir ferramentas digitais para a modelagem ou para se registrar modelos (carência da literatura e da comunidade) se tornou uma condição essencial para um estudo mais eficiente em ECOS na disciplina.

\section{Proposta de Ferramenta para o Apoio ao Ensino de ECOS}

Uma observação dos alunos foi a falta de exemplos de modelos ECOS, também coincidindo com uma carência da literatura [Coutinho et al. 2017b]. Na tentativa de minimizar essa lacuna, este trabalho propõe a criação de uma ferramenta digital para suportar o ensino de ECOS, com algumas funcionalidades iniciais.

Mesmo com necessidades de interação entre usuários, evolução de modelos, análise de plataformas centrais ou de interesse, e tudo isso de maneira acessível e automatizada, funcionalidades básicas são ainda uma carência para a comunidade de ECOS. Compreende-se que se uma ferramenta for bastante utilizada, ela tende a evoluir, refinar suas características, adicionar novas funções. Entretanto, é preciso que ela tenha uma base e comunidade forte para que esse crescimento seja natural. Para isso, uma ferramenta que seja disponível via internet, por um navegador web, facilitaria muito essa evolução e adoção de maneira mais generalizada.

Uma funcionalidade essencial para esta ferramenta é o cadastro de modelos de ECOS. Esse cadastro seria composto por alguns atributos para a identificação e descrição do modelo, que seriam: nome, descrição, fonte e autor. Adicionalmente, um modelo pode pertencer a categorias, como contexto educacional, jogos, sistemas corporativos, e esse atributo também é interessante para um ECOS. Como uma deficiência da comunidade é a disponibilização de modelos de ECOS, a ferramenta deve disponibilizar a consulta desses modelos. E funcionalidades de filtragem também seriam de muita utilidade, para facilitar as buscas específicas. Uma funcionalidade que provenha algum nível de controle de acesso é necessária, o que leva a um cadastro de usuários, o qual detém o poder de cadastrar modelos e alterar apenas os modelos os quais foram cadastrados pelo próprio usuário identificado na ferramenta. Uma última funcionalidade é em relação à qualidade dos modelos. Nem toda imagem é um modelo de ECOS, e nem todos os modelos são úteis, sendo necessário alguma forma de controle de qualidade.

Em sendo desenvolvida, esta ferramenta traria diversos benefícios para a comunidade de Engenharia de Software e de ECOS, tais como: base de modelos de ECOS, acesso fácil pela internet, difusão dos princípios de ECOS, apoio à comunidade de ECOS, e auxílio no ensino de ECOS em Engenharia de Software. A Figura 5 apresenta um esboço de uma possível aplicação web, com uma opção de tela inicial 
VII Congresso Brasileiro de Informática na Educação (CBIE 2018)

Anais do XXIV Workshop de Informática na Escola (WIE 2018)

onde qualquer pessoa pode visualizar um modelo de ECOS e seus detalhes, uma tela de usuário identificado no sistema, onde ele pode adicionar, alterar e remover modelos, e telas para o cadastro do usuário, cadastro de categorias e controle de qualidade.

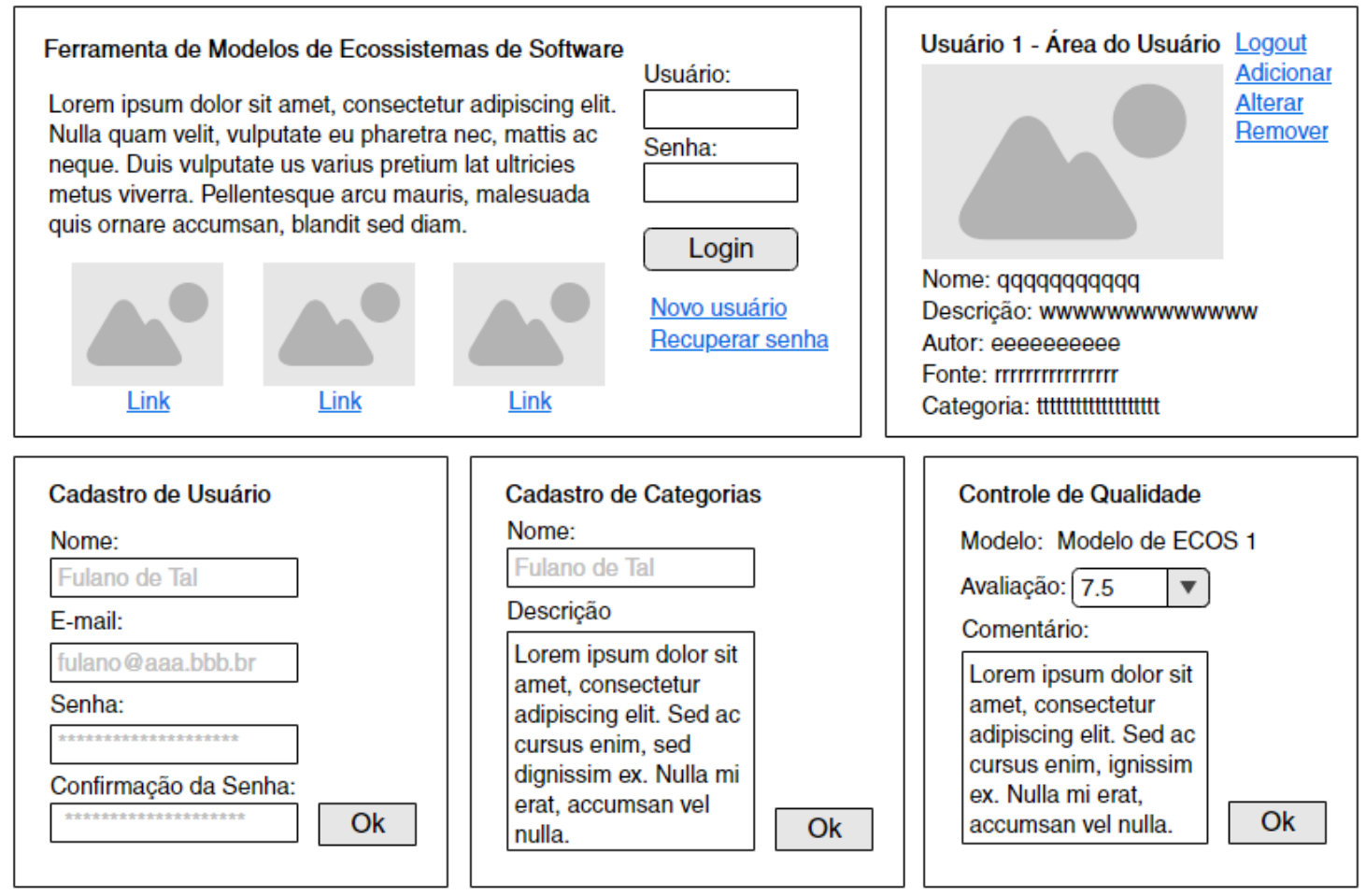

Figura 5. Mockup de telas preliminar de uma aplicação de suporte a ECOS

\section{Conclusões e Trabalhos Futuros}

Esse trabalho apresentou um relato do ensino de ECOS em um curso de graduação, na disciplina Engenharia de Software. Conceitos relevantes de ECOS foram apresentados à turma e exemplos de ECOS com base na visão dos alunos foram desenvolvidos. De maneira geral, o retorno dos alunos no assunto foi satisfatório, gerando também a proposta de uma aplicação para suportar o ensino de ECOS.

Para a próxima edição da disciplina de Engenharia de Software planeja-se aprofundar ainda mais ECOS. A intenção é trazer o conceito mais para o início do semestre, para que aos poucos os alunos percebam seus elementos e que possam refinar modelos, identificando melhorias para tanto a plataforma central quanto para o ecossistema como um todo. Além disso, a utilização de ferramentas de modelagem é essencial para uma melhor compreensão e visão geral de um ECOS. E com o desenvolvimento da ferramenta proposta, as aulas terão um caráter mais prático, podendo o estudo de ECOS ser também levado para fora da sala de aula, reforçando inclusive o uso das Tecnologias Digitais de Informação e Comunicação (TDIC).

\section{Referências}

Boucharas, V., Jansen, S., Brinkkemper, S. (2009) Formalizing software ecosystem modeling, in Proceedings of the 1st International Workshop on Open Component 
VII Congresso Brasileiro de Informática na Educação (CBIE 2018)

Anais do XXIV Workshop de Informática na Escola (WIE 2018)

Ecosystems - ACM SIGSOFT Symposium on the Foundations of Software Engineering (FSE), pp. 41-50, Amsterdam, The Netherlands, August.

Costa, G., Silva, F., Santos, R., Werner, C., Oliveira, T. (2013) From Applications to a Software Ecosystem Platform: An Exploratory Study, in Proceedings of the 5th ACM International Conference on Management of Emergent Digital EcoSystems (MEDES), 2013, pp. 9-16, Neumünster Abbey, Luxembourg, October.

Coutinho, E., Moreira, L., Sarmento, W. (2013) Maat - sistema de avaliação de alunos e tutores para um ambiente virtual de aprendizagem, in IX Simpósio Brasileiro de Sistemas de Informação ao (SBSI2013), João Pessoa.

Coutinho, E. F., Gomes, G. A. M., e Junior, A. J. M. L. (2016). Applying design thinking in disciplines of systems development. In 8th Euro American Conference on Telematics and Information Systems (EATIS2016).

Coutinho, E. F., Santos, I., M. Bezerra, C. I. (2017a) A software ecosystem for a virtual learning environment: Solar seco. In 2017 IEEE/ACM Joint 5th International Workshop on Software Engineering for Systems-of-Systems and 11th Workshop on Distributed Software Development, Software Ecosystems and Systems-of-Systems (JSOS), pages 41\{47, May 2017.

Coutinho, E. F., Viana, D., Santos, R. P. (2017b) An exploratory study on the need for modeling software ecosystems: The case of solar seco. In 2017 IEEE/ACM 9th International Workshop on Modelling in Software Engineering (MiSE).

Jansen, S., Brinkkemper, S., e Finkelstein, A. (2009a). Business network management as a survival strategy: A tale of two software ecosystems. In Proceedings of the First International Workshop on Software Ecosystems, 11th International Conference on Software Reuse, pages 34-48.

Jansen, S., Finkelstein, A., Brinkkemper, S. (2009b) A Sense of Community: A Research Agenda for Software Ecosystems, in Proceedings of the 31st International Conference on Software Engineering (ICSE), New and Emerging Research Track, 2009, pp. 187-190, Vancouver, Canada.

Jorge, F. F., Bezerra, C. I. M., Coutinho, E. F., Monteiro, J. M., e Andrade, R. M. C. (2015). A evolução do jogo itestlearning para o ensino das atividades de execução de testes de software. In XX Conferência Internacional sobre Informática na Educação (TISE 2015) - Nuevas Ideas en Informática Educativa TISE 2015.

Manikas, K., Hansen, K. M. (2013) Software Ecosystems - A Systematic Literature Review”, Journal of Systems and Software, v. 86, n. 5 (May), pp. 1294-1306.

Messerschmitt, D. e Szyperski, C. (2003). Software Ecosystem: Understanding an Indispensable Technology and Industry. The MIT Press, 1 edition.

Portela, C., Vasconcelos, A., Oliveira, S. R. B. (2017) Um Modelo Iterativo para o Ensino de Engenharia de Software Baseado em Abordagens Focadas no Aluno, Anais do XXVIII Simpósio Brasileiro de Informática na Educação (SBIE 2017).

Santos, R. E. S., Magalhães, C. V. C., Correia-Neto, J. S., Vilar, G. (2014) Ensino guiado por estudo dirigido: uma experiência no contexto da Engenharia de Software, Anais do XX Workshop de Informática na Escola (WIE 2014). 